\title{
PREDICTING DISENGAGEMENT OF JUDICIAL PROCEEDINGS BY \\ FEMALE VICTIMS OF INTIMATE PARTNER VIOLENCE IN SPAIN: A SYSTEMATIC REPLICATION WITH PROSPECTIVE DATA
}

María García-Jiménez, University of Seville, mgarciaj@us.es

María Jesús Cala-Carrillo, University of Seville, mjcala@us.es

María Eva Trigo, University of Seville, trigo@us.es

\section{Corresponding author}

María García-Jiménez, University of Seville. Department of Experimental Psychology, C/ Camilo José Cela s/n, 41018, Seville, Spain. e-mail: mgarciaj@us.es. Authors' note

The authors would like to thank the anonymous reviewers for their valuable comments, and Lara Murvartian and Jose A. Leal for the language revision of the manuscript.

Funding

This research has been supported by the Department for Social Welfare and Equality of the Andalusian Regional Government (1071/0453), by the Department of Justice of the Andalusian Regional Government (2105/0453), and by the Ministry of Education, Culture and Sport of the Spanish Government to the first author (file FPU15/00373). $\underline{\text { Keywords }}$ Abandon prosecution, legal proceedings, gender violence, prospective study, logistic regression model. 


\begin{abstract}
This paper examines which variables predict disengagement of legal proceedings by victims of intimate partner violence in the first steps of the Spanish judicial process. We replicated a previous retrospective study with a prospective sample of 393 women. The relationships of socio-demographic, emotional, motivational, and psychological variables with procedural withdrawals were analyzed. We developed a binary logistic regression model that predicts disengagement with two variables: the contact with the abuser and the interaction between this contact and the thought of going back with him. Interesting differences between the current and the retrospective study were found. Results are discussed extensively in the conclusions.
\end{abstract}


Intimate partner violence (IPV) remains a major social problem. This is confirmed by official data on the number of women affected worldwide (World Health Organization [WHO], 2013). Although compared to other European countries, such as Denmark or Finland, Spain has the lowest levels of sexual or physical IPV, at 13\% according to the European Union Agency for Fundamental Rights (2014), it stands out as a country in which $71.4 \%$ of female victims have never reported their aggressor (Government Delegation for Gender Violence [GDGV $\left.\left.{ }^{1}\right], 2015\right)$. This percentage is also consistent with the data provided by the European Union (2014). Likewise, 10.41\% of Spanish women renounce continuance of the judicial procedure by using article 416 of the Criminal Procedure Code, (General Council of the Judiciary $\left[\mathrm{GCJ}^{2}\right], 2017$ ), which refers to the exemption from the obligation to testify against the partner or ex-partner. However, the percentage of renunciations is higher, at $20.9 \%$, if it includes women who dropped out of court proceedings even before they were able to exercise this right at the time of the trial (GDGV, 2015).

In Spain a victim may leave the legal system by dropping the charges at any moment of the procedure, or by denying to testify against their partner when ratifying the complaint, or at trial, benefiting from the article 416 of the Criminal Procedure Code. Consistent with other papers on the field in Spain's literature (e.g. Vázquez, Rivas, Suarez, \& Panadero, 2018), in this work we assume different terms such as renunciation, disengagement from legal proceedings, retraction, drop charges, abandon prosecution, or refuse to continue in the legal system, among others that are understood as a victim's decision to withdraw from legal action. All these terms used in the text reflect the object of interest in this work: women leaving the judicial system once it has already been filed a complaint against the (ex)partner.

In specialized courts in intimate violence against women in Spain, women may act as a private accusation, but being a public crime, prosecutors have the duty to 
proceed as a public accusation. In this regard, women's decision to drop charges or not to cooperate with prosecution does not release prosecutors from its obligation to proceed when there is sufficient evidence of the crime, as stated by the Organic Act 1/2004 of 28 November on comprehensive protection measures against gender-based violence. However, the absence of victim cooperation constitutes a worldwide challenge (Sleath \& Smith, 2017) because it hinders the investigation when the victim is the only witness, often leading to dismiss the proceedings (Erice, 2007).

Various studies have addressed the study of possible sociodemographic and psychological variables that explain why women are reluctant to continue prosecution for IPV. It seems that economic dependence on the aggressor and the lack of tangible support when a woman breaks up the relationship could determine the decision to drop charges (Cerulli et al., 2014; Goodman, Bennett, \& Dutton, 1999). The educational level and having children are not variables clearly related with the withdrawal of prosecution since some studies have shown their relevance (e.g. Goodman et al., 1999) and others have not (e.g. Cala, Trigo, \& Saavedra, 2016; Sleath \& Smith, 2017). The fact that sometimes having children eases women to withdraw and sometimes does not may be justified by the results in Morgan and Coombes (2016), who found that mothers' involvement in legal proceedings against their (ex)partners is affected by children safety regarding complex family relationships, further abuse of children, or custody issues. The country of origin often constitutes a handicap as well and generates a situation of greater vulnerability. Migrant women may have failed to master the language, be unaware of their rights, and find it more difficult to understand the functioning of the judicial system and to have knowledge of and access to aid resources (Bennett, Goodman, \& Dutton, 1999; Herman, 2003). In the light of this, the culture of origin may also prevent women from cooperating with prosecution when they weight the impact in terms of religion, traditions, and community effects (Latta \& 
Goodman, 2005; as cited in Epstein \& Goodman, 2013), and because cultural beliefs can influence their response to victimization (Sabina, Cuevas, \& Schally, 2012).

Other factors that have shown to be determinant to women's disengagement from legal action have to do with judicial variables, like not being granted a protection order (e.g. García-Jiménez, Cala, Trigo, \& de la Mata, 2018), not feeling well supported by their lawyers, or when professionals' response to victims not only does affect women's experiences in the legal system (e.g. García-Jiménez et al., 2018; Goodman, Fauci, Sullivan, DiGiovanni, \& Wilson, 2016; Xie \& Lynch, 2017), but also may incur secondary victimization (Cubells \& Calsamiglia, 2018; Laing, 2017). The functioning of the judicial system can also affect women's decision to renounce, especially because the pace of the procedure is generally not adjusted to women's processes of recovery (Cubells \& Calsamiglia, 2018). Furthermore, the type of violence may be hindering going forward in the process. For instance, psychological violence tends to be difficult to be evidenced (Cubells \& Calsamiglia, 2018), which may discourage the victim from continuing.

From a psychological perspective, it is needed to mention the importance that these women give to their relationship and their difficulty in breaking away also carries a lot of weight (Walker, 2017). Breaking up the relationship is an arduous process that requires women to stop loving the partner and stop harboring the hope that he will change (Landenburger, 1989) since being attached to the perpetrator tends to prevent women from completing the protective order process (Zoellner et al., 2000). In addition, they often feel guilty about potential consequences after having denounced their partner, such as his feasible imprisonment (Bennett et al., 1999; Fischer \& Rose, 1995). Feelings of guilt in victims of IPV during the judicial system are significantly more frequent than in victims of other kinds of violent crimes (Buzawa, Buzawa, \& 
Stark, 2017), and would also explain their decision to leave the judicial system (Cala et al., 2016).

Numerous studies have found that not all women seeking help want to initiate criminal proceedings against their partners, and not always seek punishment for the aggressor (Ford, 1983; Gillis et al., 2006; Hoyle \& Sanders, 2000). These women may feel that they have given an exaggerated answer to their problem with the complaint because their only intention was to warn the abuser about the consequences of his actions (Hoyle \& Sanders, 2000). It must be added that, since women find themselves immersed in the cycle of violence, it is understandable behavior to renounce during the stage labelled by Walker (2017) as the honeymoon, in accordance with the cooling-off period after initiating prosecution when the woman may change her mind as a consequence of reevaluating her alternatives (Ford, 1983).

Finally, fear of reprisal or retaliation from the abuser or his friends can be paralyzing and may prevent collaboration in the judicial process (Buzawa et al., 2017; Cerulli et al., 2014; Sleath \& Smith, 2017), but it can sometimes facilitate a woman's adherence to the judicial system when she feels the need for protection (Hoyle \& Sanders, 2000), especially when children are exposed to direct and severe violence (Goodman et al., 1999).

Many of the cited papers that propose reasons for abandoning the judicial procedure are qualitative studies, which have hindered access to larger samples. Others have not included women residing in Spain, an important consideration to our knowledge when providing answers adjusted to the women's specific needs according to the singularities of each cultural context or country.

In view of these limitations, a recent quantitative study by Cala et al. (2016) with a large sample of women from the region of Andalusia (Spain), showed interesting relationships between psychological and emotional variables and the 
decision regarding whether to continue judicial proceedings. These authors developed a logistic regression model capable of accurately predicting $80.2 \%$ of renunciations with four factors: whether or not women received psychological support; the frequency of contact maintained with the aggressor; whether or not they considered returning to the abuser during the judicial procedure; and the feeling of guilt.

However, Cala et al. (2016) used retrospective data: the participants had already completed the judicial procedure and it was known whether or not they had abandoned it when they answered the questionnaire. Thus, from the predictive model they developed one could ask the following question: would the most important predictive variables for this model be the same for those who have not yet completed the judicial procedure and for those who are at the earliest stages of this procedure? In this sense, experiences related to the judicial system may have had a different impact on women who have already come a long way in it, in contrast to those taking their first steps in the judicial system. In fact, for women who answered retrospectively, emotions could have been less intensely evoked or even removed from memory in an effort to put past suffering behind them and start a new life. It is possible that the passage of women through the judicial system contributed towards a reworking of their experiences on an emotional and psychological level. In this way, the predictive model based on their experiences would not serve to predict the behavior of women who are starting the judicial itinerary. An accurate prediction would be crucial for any intervention before women could be tempted to leave the judicial process. It would also allow measures to be taken to enable them to move forward, by keeping them under the protection of security measures that would otherwise be greatly reduced on renunciation. Knowledge of the reasons for disengagement will help to legal practitioners avoid the frustration and help to better understand the reasoning behind these seemingly paradoxical decisions made by women (Goodman et al., 1999), 
thereby preventing them from being blamed for taking an unexpected course of action. All this justifies the need for the creation of a model that also includes the experience of women who are starting their journey in the judicial system.

This work, therefore, strives to analyze whether, and in what sense, the predictor variables of the renunciations proposed by Cala et al. (2016) differ when considering prospective data. In this regard, the first aim is to replicate the original study by using a sample of women who had not completed the judicial procedure and of whom it remained unknown whether or not they would renounce when they completed the questionnaire. As replication of a previous study, the variables to be analyzed in the current work will be those in the same blocks of predictors raised in Cala et al. (2016): socio-demographic, psychological, emotional, and motivational variables. This replication will include: a) the analysis of relations between each independent variable and leaving the legal proceedings; and b) the development of a binary logistic regression model to predict disengagement using prospective data.

The second aim of this paper is to compare the prospective data in this study and the retrospective data from Cala et al. (2016) in women's answers to the questions of the main predictive variables found in Cala et al. (2016). We expect first that the resultant predictive model will differ from the original, and second, that this difference will be due to variations in the way women responded the questions in comparison with women in Cala et al. (2016), since each sample answered the questionnaire in different moments of the legal proceedings (when they had already finished it or when they were still immersed in it).

\section{METHOD}

\section{$\underline{\text { Participants }}$}

Figure 1 shows a flowchart to illustrate the process of data collection. Although 779 women were asked to participate in the study, only 763 accepted, having a total of 
806 questionnaires $(n=43$ were double cases of women who had answered by two open procedures, but we considered only the most current case for analysis purposes). Out of the 763 women, 345 had already completed the judicial procedure at the time of completing the questionnaire (retrospective sample considered in Cala et al., 2016). The remaining 418 women were in an open procedure and it remained unknown whether they would renounce or not (prospective data). This information could be accessed once women finished the legal proceedings, having 25 lost cases for the dependent variable because it was not possible to locate these women, and this information did not appear in their court file. The sample for the replication study, therefore, finally consisted of 393 women.

For the second aim of the study, data from these 393 women and from the 345 women from Cala et al. (2016) were analyzed $(n=738)$ to compare the differences in the responses to the questionnaire between the prospective and the retrospective samples regarding the four predictive variables in Cala et al.'s work (2016).

Figure 1 about here

The 763 women were accessed for convenience and accessibility, being recruited from different services in Andalusia (Spain): Victim Assistance Service of Andalusia [VASA $]^{3}$, Women's Information Centers, shelters, and different associations for IPV assistance. In this study, all women $(n=393)$ were users of the VASA ( $n=61$ were at the same time users of Women's Information Centers) in Seville $(n=270)$ and Granada $(n=123)$, but there were no women from shelters or other such associations. The VASA provides women with psychological, social, and legal accompaniment and guidance during the legal process, but does not provide psychological treatment. VASA offices are located in the same building than IPV 
specialized courts in the capitals of provinces in Andalusia, but not in rural areas. However, any woman facing IPV legal proceedings can use this service at any moment, no matter where she lives.

Regarding the participants in the current study, their ages $(n=388)$ ranged from 15 to 78 years old $(M=34.23, S D=10.92)$. Their average number of children $(n$ $=388)$ was $1.42(S D=1.24)$, and their average income $(n=381)$ was 391.52 euros per month $(S D=448.77)$, although $67.7 \%$ received less than $€ 500$ per month. The level of studies ( $n=393)$ was varied: $17 \%$ of women had no studies, $68.2 \%$ had compulsory studies or vocational training, and $14.8 \%$ had a baccalaureate or university degree. As for the country of origin $(n=393), 85.2 \%$ of women were Spanish, $8.7 \%$ were from Latin American countries, and 6.1\% came from non-Spanish-speaking countries. At the time of data collection, all the 393 women were involved in the judicial proceedings for IPV in the Andalusian Courts for Violence against Women. The $67.4 \%$ of women found themselves in the first 72 hours after the complaint, $17.9 \%$ were in the rapid-trial phase, and $12.4 \%$ were in the stage of judicial investigation. For the current study, it was not possible to know the moment when women abandoned prosecution for those who finally did it since this information was not available in the women's court files.

\section{$\underline{\text { Materials }}$}

The participants completed the same ad hoc questionnaire as that given to the women in the study by Cala et al. (2016), which was based on the previous study by Cala et al. (2012). The Appendix includes the basic format of 19 questions analyzed in this study, taken from the complete questionnaire. The questionnaire was developed following a process to maximize content validity: it was conducted an in-depth review of the literature about the topic, interviews with 21 professionals from different services in Andalusia, and with 14 women victims of IPV to detect any variable 
related to disengagement by content saturation. The first version of the questionnaire was evaluated by an external committee of 8 experts in the topic and professionals in psychological assistance to IPV victims. The resultant version was piloted by the research team with women victims in order to ensure that no question led to doubts nor ambiguity. It was not conducted a study of the reliability of the questionnaire for practical and ethical conditions, because of the length of the questionnaire and its sensitive content.

The questions focused on women's perceptions and experiences. Those that referred to data or facts (number of children, country of origin, etc.) were multiplechoice, while questions related to opinions, emotions, experiences, and beliefs were Likert-type items where the response rating ranged from 0 to 10 (see Appendix). Open-ended questions were coded as in Cala et al. (2016) (see Appendix). Information about some variables such as the severity of violence, the result of the legal process, or the consequences for the aggressor could not be obtained, since this information did not depend on women's answers, and the judicial system did not authorize the access to this information through the court files.

The instruments were individually administered and completed by the VASA staff during their accompaniment services. The duration of the application and the order of the questions were variable. Each woman was assigned an identification number during the first data collection process for monitoring purposes.

\section{$\underline{\text { Procedure }}$}

The complete data set $(N=763)$ was collected for a three-month period in 2011 (see Figure 1). Both retrospective and prospective data were collected identically for the entire sample: we first submitted the project to obtain ethical approval after being reviewed by the Department for Equality and Social Welfare and the Department of Justice of the Andalusian Regional Government, as well as the Research Foundation 
of the University of Seville, and to obtain the approval of directors of services (VASA, Women's Information Centers, shelters, and associations) before contacting the women users of these services. Service professionals were instructed to administer the questionnaires and did it after women were informed about the general study purposes, confidentiality, and that their responses would not affect or interfere in the results of the legal proceedings. Neither the professionals nor the women knew the hypotheses of the project. The main difference regarding the procedure for the current study was that we collected the dependent variable for the prospective data in 2014, once the legal proceedings had finished and it was possible to know whether a woman had finally disengaged or not. It was necessary to rely on the participation of VASA professionals for this second phase of the data collection, as they had access to this specific information through women's court registries. Each woman was identified using the locator code assigned and only when it was impossible to obtain the data of interest through the registries and databases of the Judicial System, women were contacted by telephone $(n=47)$.

\section{$\underline{\text { Data Analysis }}$}

In order to study the relationship between categorical independent variables and the dichotomous dependent variable (whether to abandon prosecution or not), Pearson's Chi-square test was used with the contingency coefficient as the effect size index. We performed the post hoc study of standardized residuals in contingency tables with more than 1 degree of freedom. The groups showing differences were those whose residuals were over the expected $Z$ for the adjusted significance level (.05 / number of cells examined). For the quantitative variables, we developed betweengroup ANOVA (with Snedecor's or Welch's $F$ test), whereby $R^{2}$ was taken as the effect size index. The ordinal variables, all of them with more than 5 values (Likert 
scale $0-10$ ), were considered as quantitative, checking the assumptions required for the parametric tests applied to these type of variables.

Subsequently, the binary logistic regression model was developed to replicate that of Cala et al. (2016). Finally, ANOVA and Chi-squared tests were used to compare how women responded to the questionnaire in the two studies regarding the predictive variables in Cala et al. (2016).

The level of statistical significance was $\alpha=.05$ and the effect size was assessed as small $\left(R^{2}=.01, \Phi=.10\right)$, medium $\left(R^{2}=.06, \Phi=.30\right)$, or large $\left(R^{2}=.14, \Phi=.50\right)$, in accordance with Cohen (1988). Statistical power, calculated post hoc by the G*Power 3.0 program for a medium effect size (Faul, Erdfelder, Lang, \& Bushner, 2007), was equal to or greater than .99 for all the test developed. Data analysis was performed using SPSS 20.

\section{RESULTS}

A first descriptive study showed that 308 women continued with the judicial procedure $(78.1 \%)$ and 85 women $(21.9 \%)$ disengaged. Descriptive data for all the variables analyzed for women that disengaged and women that did not are available in this $\underline{\text { link. }}$

\section{$\underline{\text { Relationship Between Each Independent Variable And Disengagement }}$}

The results for the ANOVA and Chi-squared tests are described in groups of variables. For the socio-demographic data (educational level, country of origin, ruralurban residence, number of children, age, and monthly income), there was no relationship between the decision to abandon prosecution and most socio-demographic variables, except for: the residence of women, $\chi^{2}(1, N=391)=11.10, p=.001, \Phi=$ .17 ; and the number of children, Welch's $F(1,158.75)=4.15, p=.043, R^{2}=.01$. Thus, women who belonged to urban areas renounced more $(25.7 \%)$ than women from rural areas $(9.5 \%)$, and women who renounced had fewer children $(M=1.23, S D=$ 
$0.95)$ than those who did not renounce $(M=1.48, S D=1.16)$. Both factors reached statistical significance, but the effect size found did not reach the medium level.

No statistically significant relationship was found between the renunciations and any of the psychological variables (psychological support received, time in days of psychological support received, or support received from family and friends) with small effect sizes.

Abandonment of the procedure was not significantly related to most of the emotional variables (Table 1). Only the contact with the (ex)partner showed differences between women who disengaged with respect to those who did not and reached a medium effect size. The analysis of standardized residuals showed that the difference was found between the percentage of women who disengaged when contact was occasional (30.3\%) compared to the percentage of women who disengaged but without contact with the aggressor (6.2\%). Regarding the type of contact $(n=44)$, it was primarily through the children (54\%) and by telephone (48\%), rather than face-toface contact $(30.4 \%)$.

Table 1 about here

Finally, Table 2 shows that for the variables of motivational type, only the relationship between the expectation of separating when the complaint was filed and the act of renouncing was significant. The percentage of renunciations was lower in women who expected to be separated (15.9\%), compared to those who did not have this expectation (25.6\%), although the effect size was small.

Table 2 about here 


\section{Validation Of The Statistical Model With The New Data}

For the replication of the binary logistic regression model, the same variables were assumed as those that were taken in Cala et al. (2016): psychological support, contact with the aggressor, consideration of returning to the aggressor, and feelings of guilt. The decision to include the same variables as the original model, and not those that would have obtained a statistical significance and at least a medium effect size as established by the criterion indicated by Cala et al. (2016), is due to the fact that none of the variables except that of contact with the aggressor fulfilled this criterion, which would, therefore, give rise to a predictive model constituted by a single variable and would dismiss the effect of the other variables and their interactions. The interaction between all the independent variables was tested and it resulted that the relationship between the contact with the aggressor and the consideration of going back with him had a significant effect and reached close to a medium effect size, $\chi^{2}(2, N=181)=$ $13.54, p=.001, \Phi=.26$. The study of standardized residuals showed that the highest percentage of women who stated that they considered going back with him was among those having frequent contact $(33.3 \%)$, in contrast to those who maintained no type of contact $(6.2 \%)$ or had only occasional contact with the (ex)partner (5.3\%). Thus, it was necessary to introduce such interaction into the model.

Table 3 shows the results obtained from the binary logistic regression analysis using a forward-step method and the same orthogonal contrasts than in Cala et al. (2016) for the variables of psychological support from social services and of contact with the aggressor after the complaint had been filed. The tolerance index for the variables introduced in the model was .97 and the VIF (Variance Inflation Factor) was 1.03, and hence there are no problems of multicollinearity. A forward-step method was used which, by means of the probability index, would help to obtain the most parsimonious model possible. The model's likelihood ratio declined significantly from 
the deviation from the observed data, and the Hosmer-Lemeshow statistic revealed no significant differences between observations and predictions from the statistical model. The two variables that were selected to predict the renunciations were those of contact with the aggressor and the interaction between this contact with the aggressor and the consideration of returning to him. From the odds ratio column (OR), the act of maintaining contact (albeit frequent or occasional) with the abuser, in contrast with no contact maintained, multiplied the risk of renouncing by 7.93 , while this risk multiplied by 4.26 when contact was frequent, in contrast with no contact. On the other hand, the interaction between the frequency of contact and the consideration of returning to the aggressor showed that when the women think about going back with their partner, the relationship between renunciations and frequent contact is significantly lower.

Table 3 about here

Finally, in the classification of cases with this model, and taking cases with probabilities higher than .20 as renunciations, the model has a specificity of $74.4 \%$ and a sensitivity of $76.2 \%$. Overall, the model accurately predicts $74.7 \%$ of cases.

\section{Comparison Of The Response Of Women In Each Of The Two Studies}

Finally, data from the retrospective study $(n=345)$ and from the current one (prospective, $n=393$ ) were analyzed to compare the responses to the questionnaire by the women in both studies, independently from having disengaged or not, and regarding the variables in the retrospective predictive model in Cala et al. (2016): the psychological support received (via social services, health services, or none); the contact with the aggressor (none, occasional, or frequent); the consideration of returning to the aggressor (no or yes); and the feeling of guilt (self-rated $0-10$ ). 
We found differences in the psychological support received by the women in both studies, $\chi^{2}(2, N=708)=60.14, p<001$, with an effect size close to medium level, $\Phi=.28$. The study of standardized residuals showed that a significantly lower percentage of women in the current study $(24.70 \%)$ had received psychological support from Social Services than women in the retrospective study (38.90\%), while $75.30 \%$ compared to $52.20 \%$ stated that they received no psychological support.

As for the contact with the aggressor, the differences found were statistically significant and with an effect size close to medium, $\chi^{2}(2, N=485)=40.44, p<.001$, $\Phi=.28$. The study of standardized residuals showed that $8.80 \%$ of the women in the current study maintained frequent contact, compared to of the $25.20 \%$ women in the retrospective study and $70.60 \%$ compared to $40.60 \%$ maintained no contact at all.

In the retrospective study, $33.30 \%$ of the women admitted to having considered restarting the relationship, whereas in this new study only $6.40 \%$ of them acknowledged this idea. These differences were statistically significant and with a medium effect size, $\chi^{2}(1, N=730)=85.26, p<.001, \Phi=.32$.

We also found statistically significant differences in how they rated their feelings of guilt, although the effect size did not reach a medium level, Welch's $F$ $(1,674.44)=18.83, p<.001, R^{2}=.03$. Women in this prospective study felt less guilty $(M=3.48, S D=3.73)$ than those in the retrospective study $(M=4.76, S D=4.09)$.

\section{DISCUSSION}

The retrospective study by Cala et al. (2016) was replicated in order to ascertain whether there would be differences in the predictive variables regards renouncing the procedure, depending on whether the women victims of IPV had already finalized the legal proceedings against their (ex)partner or not when they answered the questionnaires. Differences were found in the individual relationships between the dependent variable (abandon prosecution) and independent variables. The 
replication of the binary logistic regression model of Cala et al. (2016) also showed differences in the number of predictors and the importance of each one in the prediction of the variable of interest. These differences are largely explained by the results of the comparison of the way women answered when it was already known whether or not they had renounced compared to when they were in the early stages of the judicial procedure at the time that this fact remained unknown. This comparison between the prospective and retrospective study constituted the second objective of this study. The differences between studies in the essential variables for the predictive model of Cala et al. (2016) will be highlighted.

Only $21.9 \%$ of women in this study dropped charges. This shows that most women achieved the end of the procedure. However, we should not consider that disengagement means that women do not try to end the violence actively, but they are using different strategies to achieve it. It seems reasonable to say that the decision regarding whether or not to renounce a judicial procedure may be predicted by different variables depending on whether the women answered the questionnaire once the procedure was completed or whether they did so shortly after initiating it. That is, the variables for the prediction are different when the prediction is made at one time or another in the judicial procedure. The majority of women whose data was analyzed in this study found themselves in the first 72 hours of the judicial procedure (67.4\%) or in the rapid-trial phase (17.9\%), which are very early stages of the Spanish judicial process. This may point towards expectations and emotions that may not be the same for those who have just reported the violence as for those who have been immersed in the judicial system for months or years or have even finished the whole procedure.

We now explain the differences found between the study by Cala et al. (2016) and this one in terms of the different blocks of variables analyzed. 
From a general perspective, it appears that the results from this study indicate that no socio-demographic variable acquires sufficient relevance to explain the renunciations. The homogeneity of the sample regarding variables like educational level and economic income could explain the absence of differences between the group of women who renounced and those who did not.

The educational level was not a conclusive variable in the study by Cala et al. (2016) and in this sample, it remains largely irrelevant in the prediction of renunciation. However, we have to mention that in the current study the majority of women $(68.2 \%)$ had only mandatory studies. Similarly, economic income was also insignificantly related to the act of disengagement. Although economic independence from the aggressor may facilitate the end of the relationship of violence, the reporting, and the continuation of the initiated procedure (Cerulli et al., 2014; Goodman et al., 1999; Erice, 2007), as in the original work, the income level of the majority of women was less than 500 euros per month.

The country from whence the women came also remained insignificant, in contrast to the previous study. Although culture has been shown to be relevant in some studies (e.g. Sabina et al., 2012), Bennett et al. (1999) indicated that knowledge of the language, more than the country, would make a difference when deciding whether to abandon the judicial process, since its lack would hinder access to resources and information (Herman, 2003). Perhaps the fact that the majority of the women participants in the current study were Spanish and Latin American, and therefore proficient in Spanish, has prevented any of the expected differences from being found.

Finally, the fact of having children is, therefore, a largely inconclusive factor, which reinforces the lack of conclusions about the effect of having children in abandoning the procedure. While some studies have found this relationship (Goodman et al., 1999), others deny its existence (Hare, 2006; Sleath \& Smith, 2017). As 
proposed by Cala et al. (2016), having children in common with the perpetrator could lead women to continue in the judicial process when the abuse is severe since, when faced with the possibility of risk for the minors, women accelerate the breakup and ask for help (Landenburger, 1989) as long as being involved in the legal proceedings do not endanger children's safety (Morgan \& Coombes, 2016).

\section{$\underline{\text { Psychological Variables }}$}

An available resource in the region of Andalusia is the Women's Information Centers in every county, which may offer free individualized psychological treatment for women victims of any form of violence against women. However, they mainly implement group therapy interventions. A woman is referred to a group depending on her demands and/or her needs assessed by the psychologist in the center. Whether this kind of psychological support was received or not formed an essential variable in the predictive model of renunciations in the retrospective study in such a way that lacking such support multiplied the risk of disengagement by 3.37 (Cala et al., 2016). However, in this study, psychological support showed no relevance in the prediction of renunciations. These results are consistent with those of the prospective study by Goodman et al. (1999), who also found no relationship between emotional support and cooperation during court proceedings. This remarkable change in relation to the study by Cala et al. (2016) could be explained by differences in the presence or absence of psychological support for the women in either study. That is, only $24.70 \%$ of the women who found themselves in the early stages of the judicial procedure (current study) were receiving psychological support at the time of answering the questionnaire, compared to $38.9 \%$ of women who had already completed the procedure (Cala et al., 2016).

The absence of such support in the initial stages of the procedure could be due to the fact that these women were not yet aware of the psychological assistance 
services, as a consequence of a lack of information before the complaint (Camacho \& Fiftal, 2008). Another interpretation might be that the access to these resources is increased throughout the procedure because when women come into the judicial procedure then the consequences of violence may be aggravated (e.g. Cluss et al., 2006) but also because of secondary victimization (Cubells \& Calsamiglia, 2018; Laing, 2017) and the effects of the legal procedure in their well-being (Herman, 2003). Psychological support can be crucial in that it gives women tools to move forward in their lives. As López-Fuentes and Calvete (2015) found, formal social support like mental health services and group therapy helps women in their resilience process and promotes other personal factors that are important for their recovery. However, as indicated from the data, it seems that this psychological support is given especially when women have already been immersed in the judicial system for a considerable time since there were few women receiving psychological support at the beginning of the procedure. It may be that the passage through the judicial system itself, for those who have already completed it or are in the later stages, has led to secondary victimization and/or retraumatization (e.g. Laing, 2017), which in turn led them to need and demand psychological help, as stated by Herman (2003), regarding the negative impact of the legal procedure on women's mental health. This interpretation is undoubtedly of concern, since it may show that the absence of sensitized and adequate treatment by the legal operators may have a strong negative impact on the victims. Indeed, it has been shown that victims' satisfaction with the legal system affects their well-being (Kunst, Popelier, \& Varekamp, 2015).

In the same way that social support during the legal process has shown to be crucial (Bell, Perez, Goodman, \& Dutton, 2011; Ekström, 2015; Goodman et al., 1999), counting on an adequate psychological and emotional support seems to be important insofar as it helps toward rupturing the cycle of violence, and makes women 
aware of what they suffer, promote resilience (López-Fuentes \& Calvete, 2015) and, as a result, facilitates their collaboration with the judicial system (Cala et al., 2016).

\section{$\underline{\text { Emotional Variables }}$}

The only emotional variable that remained consistent with the original study was that of contact with the aggressor. Renunciations were less pronounced when women had no contact and increased when it was occasional and frequent. Despite the relevance of this variable in both prospective and retrospective studies as a predictive factor in the regression model, we have not found previous papers regarding victims' cooperation with the legal system that have focused on this factor. This raises the need for further research on this aspect in future studies.

However, it may be that contact could be an indicator of the attachment still existing towards the perpetrator. This could be explained given the relationship of frequent contact with her idea of going back with him after filing a complaint. In fact, this interaction between both variables was one relevant predictor of renunciations in the regression model, as it will be explained later in the paper. Thus, maintaining contact with the (ex)partner might be playing a double role: on the one hand, it may be pointing out the weight of that contact in the control that aggressors exercise over victims and in their decisions and actions regarding the judicial procedure by, for instance, threatening them (e.g. Cerulli et al., 2014); on the other hand, it would give additional evidence about the fact that women victims of IPV face a long and difficult process to end the relationship (Anderson \& Saunders, 2003).

In this regard, it is hardly surprising that women experience comings and goings in the relationship without completely breaking contact with their partner (Landenburger, 1989), and the cycle of violence continues and increases the likelihood of renunciation occurring at the honeymoon stage (Walker, 2017). At this point a woman may perceive that it is possible to restart the relationship because she truly 
believes that it has improved: she could have even pressed charges, not in order to terminate the relationship but to maintain it by stopping the aggression (e.g. Buzawa et al., 2017).

Regarding the consideration of returning to the perpetrator, this variable did not predict renunciation on its own, despite this was the variable with the greatest predictive power in the model by Cala et al. (2016). It is, therefore, necessary to mention several aspects. First, the interaction between contact and the consideration of going back was significant in predicting the renunciations of the resulting logistic regression model. Thus, when contact with the (ex)partner is frequent, but women have considered returning to him at any time of the procedure, the most important variable is the consideration of returning to the ex-partner, not so much the frequency of contact. These findings are in the same direction that those by Schmidt and Steury (1989), who found that continuing the relationship determined women's decision about prosecution.

Second, the absence of statistical significance as an individual independent variable for the prediction of renunciations could be explained by the fact that in this study few women admitted to having considered restarting the relationship, compared to women in the retrospective study (Cala et al., 2016). It is understandable that the lack of representativeness of women who consider returning to their partner marks an important difference between the two studies. It should be borne in mind that in this prospective study, most women answered our questions when they had just started the judicial procedure, so it may be that they were unlikely to confirm that they wanted to return to their (ex)partner in a moment when they had just armed themselves with the courage to denounce and break up the relationship, a difficult step to take in a complex process (Anderson \& Saunders, 2003; Landenburger, 1989). Secondly, for those who filed a complaint in order to change the abuser's behavior, so that he would stop 
bothering her, or as a warning sign, then social desirability could have prevented them from recognizing that they were in fact still deceived by false love. This is coherent for women in the very beginning of the recovery process who may tend to maintain the relationship (Landenburger 1989), and it would be less likely in the women surveyed in Cala et al. (2016) in which, from a retrospective view, they saw their situation as more emotionally distant and had a certainly experienced route in the judicial system. In addition, women in Cala et al. (2016) had received psychological support to a greater extent, which might have well made them easier to recognize and accept their emotions and experiences as part of their recovery (Flasch, Murray, \& Crowe, 2017).

On the other hand, the transition from a retrospective to a prospective study could have also affected other emotional factors in their relationship with disengagement: fear, concern about the partner entering prison, and feelings of guilt. In the prospective study, these variables made no difference in the decision of whether to continue the judicial procedure. This difference in outcomes in comparison with Cala et al. (2016) could be explained by the fact that, at the beginning of the procedure, women may not have even fully reflected on or experienced the outcomes of the rupture, of the complaint, or the consequences of the procedure, those for the aggressor (possible imprisonment) and for her, factors that have been proved to be related to decrease women cooperation with prosecution (e.g. Bennett et al., 1999; Cerulli et al., 2014; Fischer \& Rose, 1995). As women progress through the judicial process, fear, guilt, and concern may vary with the appearance of these possible precipitating factors. The cooling-off period that Ford (1983) proposed would support this explanation because, during these days, the woman could reconsider her options and change her decisions depending on the assessment of the alternatives.

Although fear may cause women to continue in the judicial system and perceive it as a guarantee of protection (Goodman et al., 1999; Cala et al., 2016), it 
may be that at the beginning of the procedure their level of fear remains insufficient for them to feel the need for this protection. In turn, psychological support may be significant to maintain and not underestimate the danger that women find themselves in (Hart, 1988; cited in Walker, 2017) after the breakup (Cluss et al., 2006). However, we should recall that, in this study, $75.30 \%$ of the women did not receive any psychological support at the time of answering the questionnaire.

In respect of the concern that the perpetrator could enter prison and the absence of any relationship with disengagement, in contrast with Cala et al. (2016), it is important to mention that women do not commonly go to court with the intention that their (ex)partner enters prison (e.g. Bennett et al., 1999; Ford, 1983; Gillis et al., 2006). When the women realize, as a possible result of the cooling-off period (Ford, 1983), that there is a possibility of this happening, they become aware that their expectations regarding the judicial system are not going to be fulfilled, which could lead them to drop charges (Hoyle \& Sanders, 2000). This would only happen once they are informed of what can happen throughout the entire procedure. Although Camacho and Fiftal (2008) indicated that this information is not always guaranteed, in Spain women have the right to this information at the moment they file a complaint. However, as it comes at a time of duress and great emotional lability, they might fail to assimilate this information. The absence of relation with the renunciations in the prospective sample is therefore understandable.

Regarding the victims' feelings of guilt, findings have shown a notable contrast between the two studies. This variable has not turned out to be significant in the prospective study, despite being a variable included in the predictive model of Cala et al. (2016). Similar to the two variables mentioned previously, it may be that, before the cooling-off period proposed by Ford (1983) in the first hours in which the woman approaches the judicial system, she has not yet fully reflected on having transgressed 
patriarchal norms (Barnett, 2001) and on what may involve for her the actions that she has initiated. Feelings of guilt, much more common in IPV cases than other types of crimes in court (Buzawa et al., 2017), require awareness of the consequences of our acts, so they would appear in the more advanced stages of the judicial proceedings, when women have reflected on what the complaint could lead to, and when they have begun to perceive social pressure as a victim of IPV (Buzawa et al., 2017; Cerulli et al., 2014), as might have occurred in Cala et al., 2016.

$\underline{\text { Motivational Variables }}$

Of all the variables concerning the expectations of women when they filed the complaint, only the expectation of separation was related to the renunciations. Although the descriptive data follows the direction of the findings of Cala et al. (2016), the effect size was small, and hence the results remain inconclusive. However, it is worth noting the lower percentage of women that abandoned prosecution having this expectation. This could be in line with those findings showing less disengagement when there was low contact with the (ex)partner and when restarting the relationship was not considered. This combination of the three variables seems to show a definite end of the relationship. In this regard, it would be very interesting for future research to focus on the study of the relationship between these three variables in order to better understand the independent relationships found in the current work.

On the other hand, and regarding expectations when filing a complaint, the literature bears witness to the importance of fulfilling women's expectations regarding the judicial system for them to continue in it (Erice, 2007; Ford, 1991; Hoyle \& Sanders, 2000). Other expectations such as getting protection and giving him a scare were conclusive variables related to renouncing in Cala et al.'s study (2016). To explain the differences in the findings of the current study regarding these variables, we may argue that what the women expected could have changed in line with their 
experience in judicial matters. Over time, the psychological and social support they could have received may have transformed their expectations due to the reprocessing of their experiences and awareness of what they truly expected when they entered the judicial system.

In light of the results found, the effect of the possible processes and changes during the passage of time could be assumed to be essential due to the way in which these changes affect other variables and the relation of these to the renunciations to the judicial procedure. In fact, certain variables that have held no importance in explaining the abandonment by women of court proceedings, such as the lack of effect of psychological and emotional support received, have been supported by the prospective work carried out by Goodman et al. (1999). These authors also explained in their work how having administered the questionnaire soon after the traumatic event could have interfered with the responses, given the emotional lability of the women at that time, and proposed that these responses would have been different if they had been prompted at the time of the trial.

\section{$\underline{\text { A New Predictive Model }}$}

The differences found between the retrospective and the prospective studies in terms of the individual relationships between the various variables and the abandonment of the judicial procedure have given rise to a predictive model that is both different and interesting. The current regression model, with only 2 variables, is able to accurately predict $74.7 \%$ of the cases of renunciations for women who find themselves mostly in the initial stages of the judicial process. These variables are those of: contact with the aggressor, for which the risk of renunciation is multiplied by 7.93 when it passes from no contact to occasional or frequent contact; and the interaction between frequent contact and the consideration of returning to the partner, whose OR (0.009) indicates that when women consider going back with him, the relationship 
between renunciations and contact ceases to be of importance, and vice versa. The model of Cala et al. (2016) accurately predicted $80.2 \%$ of the cases, although 4 variables were necessary.

Comparing both models, it is clear that it is essential to ascertain the moment within the judicial procedure at which renunciations are predicted in order to determine which variables carry the greatest weight in the prediction of when a woman is most likely to abandon court proceedings. The new model is highly interesting especially if one takes into account that predicting the behavior of women who have suffered violence in their relationships is useful at the beginning of or during the procedure, and not once it is over, since it is imperative to intervene as soon as possible and to take the necessary precautionary measures, which should be adapted to the situation of each woman. However, the previous model ensures certain relevant variables when women have already acquired some experience with the judicial system. This justifies the need to create an alternative model that contemplates the predictive variables independently of the moment of the procedure at which the woman finds herself.

The creation of predictive models such as the one developed here would have very important implications. Firstly, at the implementation level, the predictive algorithm could be easily developed as software available to professionals intervening with women facing legal proceedings (i.e. VASA or similar court services, Women's Information Centers, Shelters, etc.). The software itself would not only indicate a woman's likelihood of leaving the legal system but would also identify which variable(s) carries the highest weight in that predicted risk. With this information, this software would provide possible recommendations for professional performance. Thus, the implications at the professional level are clear and at the same time imply an improvement of services, more adapted to women's individual situation regardless of 
the decision they finally make, whether they continue the procedure or not. Nevertheless, despite the fact that disengagement may be predictable, this should not result in denying women's desires and needs, but to analyze their personal situation and act to better fit their interest in terms of safety and well-being. That is to say, the possibility of predicting women's disengagement from legal proceedings does not necessarily imply that the professionals prevent that decision but when this is not possible or contradicts the victim's wellbeing, they would be able to understand women's reasons to renounce. This understanding may help professionals to manage that possible frustration in the case of women's renunciation (Goodman et al., 1999).

\section{Limitations Of The Study And Conclusions}

While this study has overcome the limitation of Cala et al.'s (2016) study in terms of the lack of reliability to which retrospective self-reports may be exposed, it shares other limitations regarding the methodology, instrument, and analyses of data that should be mentioned. In relation to the sample, the homogeneity in some sociodemographic variables prevented their effects on the variable of interest from being observed. Thus, it is necessary to increase the heterogeneity in terms of different variables such as having children or educational level in order to try to clarify their relevance in dropping charges. At the same time, we should mention that data for the independent variables were collected more than five years ago. However, there have been no changes in the legislation or services functioning to date in Andalusia nor have there been any significant variations in the percentages of women who leave the procedure according to data from official reports in Spain (see on http://www.poderjudicial.es/cgpj/en/Subjects/Domestic-and-gender-violence/Activityof-the-Observatory/Statistic-Data).

On the other hand, although the instrument development followed a process that maximized content validity and minimized social desirability bias, it is needed to 
study the extent to which simple and limited answers (i.e. yes/no/I don't know) assure those complex aspects (e.g. think about returning with the partner) are well-measured. This leads to the possibility of using and/or developing validated scales instead of questionnaires to measure such complex aspects for future research. Even so, it is necessary to mention that women had the opportunity to make comments on any question in the instrument, but anyone did. An added limitation has to do with the fact that we did not develop a study of the reliability of the instrument, although this decision was made for practical and ethical reasons, as we mentioned before.

Other limitations regarding data collection and analyses that should be taken into consideration in future studies have to do with the inclusion of several variables: the severity of the events reported, the length of the legal procedure, the type of relationship with the respondent (couple or ex-partner), and the correlation with the offender's conviction, acquittal or case filed. At the same time it would be appropriate to control other variables related to the judicial procedure itself, such as whether a protection order was granted, or factors related to the performance of legal professionals, which also exert a significant impact on renunciations and in terms of secondary victimization (Ford, 1991; Herman, 2003). Since this paper focused on the replication of a previous study, we did not consider to include variables absent in the original by Cala et al. (2016). Even so, we must point out here that the majority of these mentioned variables were included in the questionnaire and have been considered in other works (e.g. García-Jiménez et al., 2018). This rise the need for a single predictive model that contemplates any type of variables studied in the different works and considering both prospective and retrospective samples of women. This newly proposed model would be potentially useful regardless of the stage in which women are in the judicial procedure by IPV. 
In summary, this study has shown that the effect of certain variables in the prediction of the abandonment of judicial proceedings in our sample is not extraneous to the moment of the judicial procedure at which victims find themselves, and a statistical model has been proposed formed of two variables with a high percentage of accuracy in the prediction. Although bearing in mind the referred limitations, with the use of a prospective methodology, it has been possible to explain the differences regarding previous works due to the changes along with the passage of time and its effects on certain variables. It also motivates research into the study of how the legal process interacts with such interesting aspects like women's recovery process (e.g. Landenburger, 1989). The goal derived from research on the topic of this work should be to make the necessary changes in the system for a real improvement in women's experience with judicial procedures but not to oblige victims to prosecute if this is not the best way to act, according to their needs and desires. 


\section{NOTES}

${ }^{1}$ In Spanish it stands for "Delegación del Gobierno para la Violencia de Género" (DGVG).

${ }^{2}$ In Spanish it stands for "Consejo General del Poder Judicial” (CGPJ).

${ }^{3}$ In Spanish it stands for "Servicio de Asistencia a Víctimas de Andalucía" (SAVA). 


\section{REFERENCES}

Anderson, D. K., \& Saunders, D. G. (2003). Leaving an abusive partner: An empirical review of predictors, the process of leaving, and psychological well-being. Trauma, Violence, \& Abuse, 4, 163-191. doi:10.1177/1524838002250769

Barnett, O. W. (2001). Why battered women do not leave, part 2: External inhibiting factors - social support and internal inhibiting factors. Trauma, Violence, \& Abuse, 2, 3-35. doi:10.1177/1524838001002001001

Bell, M. E., Perez, S., Goodman, L. A., \& Dutton, M. A. (2011). Battered women's perceptions of civil and criminal court helpfulness: The role of court outcome and process. Violence Against Women, 17, 71-88. doi:10.1177/1077801210393924

Bennett, L. E., Goodman, L. A., \& Dutton, M. A. (1999). Systemic obstacles to the criminal prosecution of a battering partner: A victim perspective. Journal of Interpersonal Violence, 14, 761-772. doi:10.1177/088626099014007006

Buzawa, E. S., Buzawa, C. G., \& Stark, E. D. (2017). Responding to domestic violence. The integration of criminal justice and human services. Thousand Oaks, C.A.: Sage.

Cala, M. J., Godoy, S., Sala, A., Trigo, E., de la Mata, M., Saavedra, F. J., ... Guerra, M. (2012). La renuncia a continuar en el procedimiento judicial en mujeres víctimas de violencia de género: Un estudio en la Comunidad Autónoma Andaluza [Disengagement from the judicial procedure in women after intimate partner violence. A study in Andalusia]. Seville, Spain: Instituto Andaluz de la Mujer.

Cala, M. J., Trigo, M. E., \& Saavedra, F. J. (2016). Women's disengagement from legal proceedings for intimate partner violence: Sociodemographic and 
psychological variables. The European Journal of Psychology Applied to Legal Context, 8, 35-42. doi:10.1016/j.ejpal.2015.10.002

Camacho, C. M., \& Fiftal, L. (2008). The significance of the victim advocate for domestic violence victims in Municipal Court. Violence and Victims, 23, 288300. doi:10.1891/0886-6708.23.3.288

Cerulli, C., Kothari, C. L., Dichter, M., Marcus, S., Wiley, J., \& Rhodes, K. V. (2014). Victim participation in intimate partner violence prosecution: Implications for safety. Violence Against Women, 20, 539-560.

doi:10.1177/1077801214535105

Cluss, P. A., Chang, J. C., Hawker, L., Hudson-Scholle, S., Dado, D., Buranosky, R., \& Goldstrohm, S. (2006). The process of change for victims of intimate partner violence: support for a psychosocial readiness model. Women's Health Issues, 16, 262-274. doi:10.1016/j.whi.2006.06.006

Cohen, J. (1988). Statistical power analysis for the behavioral sciences (2nd Ed.). Hillsdale, NJ: Lawrence Erlbaum Associates.

Consejo General del Poder Judicial. (2017). Annual report. Observatory against domestic and gender violence. Madrid, Spain. Retrieved from http://www.poderjudicial.es/cgpj/es/Temas/Violencia-domestica-y-degenero/Actividad-del-Observatorio/Datos-estadisticos/La-violencia-sobre-lamujer-en-la-estadistica-judicial---Anual-2017

Cubells, J., \& Calsamiglia, A. (2018). Do We See Victims’ Agency? Criminal Justice and Gender Violence in Spain. Critical Criminology, 26, 107-127. doi: $10.1007 / \mathrm{s} 10612-017-9379-2$

Delegación del Gobierno para la Violencia de Género. (2015). Macro survey of violence against women. Results preview. Madrid, Spain: Ministerio de Sanidad, Servicios Sociales e Igualdad. Retrieved from 
http://www.violenciagenero.msssi.gob.

es/violenciaEnCifras/macroencuesta2015/home.htm.

Ekström, V. (2015). Violence against women-social services support during legal proceedings. European Journal of Social Work, 18 661-674. doi:10.1080/13691457.2014.951600

Epstein, D., \& Goodman, L. A. (2013). Domestic violence victims' experiences in the legal system. In M. K. Miller \& B. H. Bornstein (Eds.), Stress, trauma, and wellbeing in the legal system (pp. 45-62). New York: Oxford University Press.

Erice, E. (2007). La retractación de la denunciante en la aplicación de la ley orgánica de medidas de protección integral contra la violencia de género [The retraction in the application of the Organic Act on comprehensive protection measures against gender-based violence]. Estudios de Derecho Judicial, 139, 155-188.

European Union Agency For Fundamental Rights. (2014). Violence against women: An EU-wide survey main results. Luxembourg: Publications Office of the European Union. Retrieved from http://fra.europa.eu/en/publication/2014/violence-against-women-eu-widesurvey-main-results-report.

Faul, F., Erdfelder, E., Lang, A.-G., \& Buchner, A. (2007). G*Power 3: A flexible statistical power analysis program for the social, behavioral, and biomedical sciences. Behavior Research Methods, 39, 175-191. doi:10.3758/BF03193146

Fischer, K., \& Rose, M. (1995). When “enough is enough”: Battered women's decision making around court orders of protection. Crime \& Delinquency, 41, 414-429. doi:10.1177/0011128795041004003

Flasch, P., Murray, C. E., \& Crowe, A. (2017). Overcoming abuse: A phenomenological investigation of the journey to recovery from past intimate 
partner violence. Journal of Interpersonal Violence, 32, 3373-3401.doi: $10.1177 / 0886260515599161$

Ford, D. A. (1983). Wife battery and criminal justice: A study of victim decisionmaking. Family Relations, 32 463-475. doi:10.2307/583685

Ford, D. A. (1991). Prosecution as a Victim Power Resource: A Note on Empowering Women in Violent Conjugal Relationships. Law and Society Review, 25, 313334. doi:10.2307/3053801

García-Jiménez, M., Cala, M. J., Trigo, M. E., \& De La Mata, M. L. (2018). Women's disengagement from legal proceedings for intimate partner violence in Southern Spain: Variables related to legal proceedings. Crime \& Delinquency, First Published Online. doi:10.1177/0011128718789857

Gillis, J. R., Diamond, S. L., Jebeley, P., Orekhovsky, V., Ostovich, E. M., Kristin M. Sagrati, S., \& Mandell, D. (2006). Systemic obstacles to battered women's participation in the Judicial System: When will the status quo change? Violence Against Women, 12, 1150-1168. doi:10.1177/1077801206293500

Goodman, L. A., Bennett, L. E., \& Dutton, M. A. (1999). Obstacles to victim's cooperation with the criminal prosecution of their abusers: The role of social supports. Violence and Victims, 14, 427-439. doi:10.1891/0886-6708.14.4.427

Goodman, L. A., Fauci, J. E., Sullivan, C. M., DiGiovanni, C. D., \& Wilson, J. M. (2016). Domestic violence survivors' empowerment and mental health: Exploring the role of the alliance with advocates. American Journal of Orthopsychiatry, 86, 286-296. doi:10.1037/ort0000137

Hare, S. C. (2006). What do battered women want? Victim's opinions on prosecution. Violence and Victims, 21, 611-628. doi:10.1891/0886-6708.21.5.611

Herman, J. L. (2003). The mental health of crime victims: Impact of legal intervention. Journal of Traumatic Stress, 16, 159-166. doi:10.1023/A:1022847223135 
Hoyle, C., \& Sanders, A. (2000). Police response to domestic violence: From victim choice to victim empowerment? British Journal of Criminology, 40, 14-36. doi:10.1093/bjc/40.1.14

Kunst, M., Popelier, L., \& Varekamp, E. (2015). Victim satisfaction with the Criminal Justice System and emotional recovery: A systematic and critical review of the literature. Trauma, Violence, \& Abuse, 16, 336-358.

doi:10.1177/1524838014555034

Laing, L. (2017). Secondary victimization: Domestic violence survivors navigating the family law system. Violence Against Women, 23, 1314-1335. doi:10.1177/1077801216659942

Landenburger, K. A. (1989). A process of entrapment in and recovery from an abusive relationship. Issues in Mental Health Nursing, 10, 209-227. doi:10.3109/01612848909140846

López-Fuentes, I., \& Calvete, E. (2015). Building resilience: A qualitative study of Spanish women who have suffered intimate partner violence. American Journal of Orthopsychiatry, 85, 339-351. doi:10.1037/ort0000070

Morgan, M., \& Coombes, M. (2016). Protective mothers: Women's understandings of protecting children in the context of legal interventions into intimate partner violence. The Australian Community Psychologist, 28, 59-78.

Sabina, C., Cuevas, C. A., Schally, J. L., \& Cuevas, C. A. (2012). The cultural influences on help-seeking among a national sample of victimized Latino Women. American Journal of Community Psychology, 49, 347-363. doi:10.1007/s10464-011-9462-x

Schmidt, J., \& Steury, E., H. (1989). Prosecutorial discretion in filing charges in domestic violence cases. Criminology, 27, 487-510. doi:10.1111/j.17459125.1989.tb01043.x 
Sleath, E., \& Smith, L. L. (2017). Understanding the factors that predict victim retraction in police reported allegations of intimate partner violence. Psychology of Violence, 7, 140-149. doi: 10.1037/vio0000035

Vázquez, J. J., Rivas, E., Suarez, A. C., \& Panadero, S. (2018). Retraction of complaints among female victims of intimate partner violence living in poverty in Nicaragua. International Journal of Offender Therapy and Comparative Criminology, 62, 3151-3167. doi:10.1177/0306624X17725728

Walker, L. E. (2017). The Battered Woman Syndrome ( $4^{\text {th }}$ ed.). New York, NY: Springer Publishing Company.

World Health Organization. (2013). Global and regional estimates of violence against women: prevalence and health effects of intimate partner violence and nonpartner sexual violence. World Health Organization. Retrieved from http://apps.who.int/iris/bitstream/10665/85239/1/9789241564625_eng.pdf?ua=

Xie, M., \& Lynch, J. P. (2017). The effects of arrest, reporting to the police, and victim services on intimate partner violence. Journal of Research in Crime and Delinquency, 54, 338-378. doi:10.1177/0022427816678035

Zoellner, L. A., Feeny, N. C., Alvarez, J., Watlington, C., O’Neill, M. L., \& Zager, R. (2000). Factors associated with completion of the restraining order process in female victims of partner violence. Journal of Interpersonal Violence, 15, 1081- 1099. doi:10.1177/088626000015010005 


\section{APPENDIX}

Sociodemographic variables: 1) How old are you?; 2) What is your educational level?a; 3) Where do you live? (rural/urban); 4) Country of origin (specify) ${ }^{\mathrm{b}}$; 5) How many children do you have in your care?; 6) What is your monthly income from your job, subsidy, family...? (Euros per month).

Psychosocial variables: 1) Are you receiving any psychological support? (Yes/No); 2) If yes, from what type of service? ${ }^{c}$; 3) For how long? (Years, months); 4) What is the level of support received from your family and friends $(0-10)^{\mathrm{d}}$ ?

Emotional variables: 1) How often do you have contact with your abuser after the complaint? (never/occasionally/frequently), specify the type of $\operatorname{contact}^{\mathrm{e}}$; 2) Do you think about going back with him? (Yes/No/I don't know) ${ }^{\text {f; }}$ 3) How scared are you of his reaction towards you? $(0-10)$; 4) How scared are you of his reaction towards your children? (0 - 10); 5) Do you feel your life is in danger? (0 - 10); 6) How much do you concern about the possible incarceration of him? $(0-10) ; 7)$ How guilty do you feel about what might happen to him? $(0-10) ; 8)$ Are you concerned about the lack of money/work? (0 - 10)

Motivational variables: What reason lead you to press charges? a) get him to stop abusing you; b) give him a warning; c) manage to incarcerate him; d) separate yourself from him; e) receive protection; f) others (specify).

Note: ${ }^{\text {a }}$ Educational level was merged into 3 categories: without studies, mandatory studies or vocational training, and baccalaureate or university studies; ${ }^{\mathrm{b}}$ Countries were coded as Spain, other Spanish-speaking countries and neighbours (Brazil), or nonSpanish speaking countries; ${ }^{\mathrm{c}}$ The categories in the psychological questions 1 and 2 were reduced into the following categories: without support, social services, or public health services; ${ }^{\mathrm{d}}$ Likert-scale items from $0=$ not at all, to $10=$ completely; ${ }^{\mathrm{e}}$ Type of contact was recorded as: direct, through children, telephone, family, other; ${ }^{\mathrm{f}} n=0$ women answered "I don't know", so this value was excluded from the analysis. 


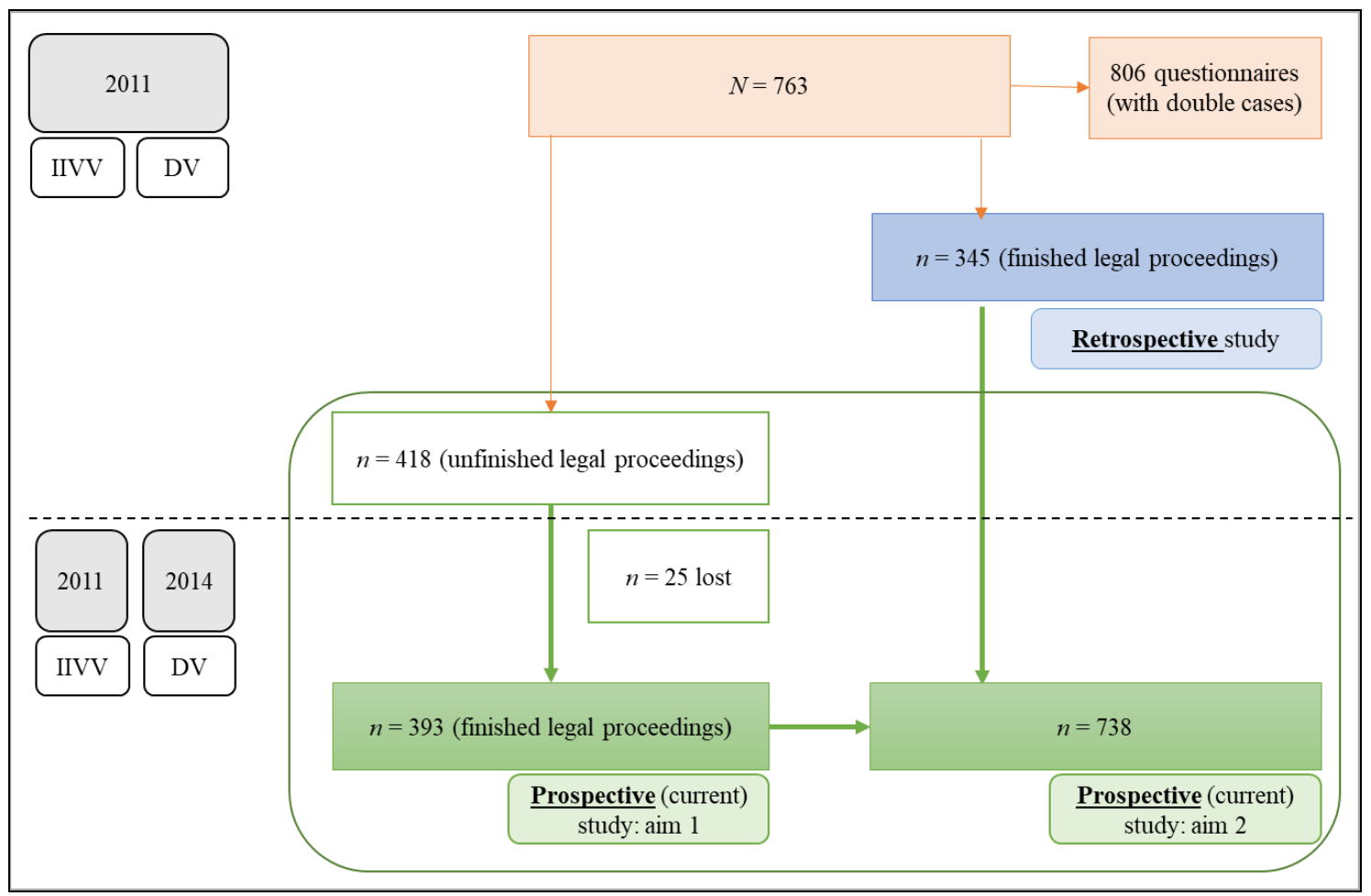

Figure 1. Flowchart of the process of data collection and sampling. The discontinuous line divides two moments of data collection: independent variables data (IIVV) were collected in 2011, the dependent variable data (DV) was known in 2011 for the retrospective study and in 2014 for the prospective study. The total of the sample is colored in orange; the information for the retrospective study by Cala et al. (2016) is colored in blue; the information for the current prospective study and aims are colored in green. 
Table 1

Results of ANOVA (Snedecor's or Welch's F) and Chi-square test for the emotional factors.

\begin{tabular}{lcccc}
\multicolumn{1}{c}{ Variable } & $F$ & $d f$ & $p$ & $R^{2}$ \\
\hline Fear towards her & 1.45 & 1,393 & .229 & $<.01$ \\
Fear towards children & .60 & 1,343 & .807 & $<.01$ \\
Feeling of being in risk & $3.92^{*}$ & 1,387 & .048 & .01 \\
Concern about prison & 1.80 & $1,17.90$ & .196 & .02 \\
Concern about money/work & .00 & 1,393 & .970 & $<.01$ \\
Guilt & .17 & 1,392 & .683 & $<.01$ \\
\hline$\quad$ Variable & $\chi^{2}$ & $d f, N$ & $p$ & $r_{\varphi}$ \\
\hline Contact with the aggressor & $16.23^{* * *}$ & 2,160 & $<.001$ & .30 \\
& & & & \\
\hline Thought of going back with him & 1.68 & 1,388 & .194 & .07 \\
\hline${ }^{*} p<.05,{ }^{* *} p<.01,{ }^{* * *} p<.001$ & & &
\end{tabular}


Table 2

Results of the Chi-square test for the motivational factors.

\begin{tabular}{lcccc}
\multicolumn{1}{c}{ Variable } & $\chi^{2}$ & $d f, N$ & $p$ & $r_{\varphi}$ \\
\hline Manage to encarcerate him & 1.26 & 1,388 & .261 & .06 \\
Stop abusing her & 3.79 & 1,387 & .051 & .09 \\
Separate from him & $5.29^{*}$ & 1,387 & .022 & .12 \\
Get protection & 2.24 & 1,387 & .134 & .08 \\
Give him a scarce & .73 & 1,387 & .394 & .04 \\
\hline${ }^{*} p<.05,{ }^{* *} p<.01,{ }^{* * *} p<.001$ & & & & \\
\hline
\end{tabular}


Table 3

Results of the binary logistic regression analysis $\left(N=150 ; n_{\text {disengaged }}=21 ; n_{n o \text { disengaged }}\right.$ $=129)$

\begin{tabular}{|c|c|c|c|c|c|}
\hline Variable & $B$ & $S E$ & $\mathrm{X}^{2}$ Wald & $d f$ & $O R$ \\
\hline Constant & -1.42 & .29 & $24.130^{* *}$ & 1 & .24 \\
\hline Contact with the aggressor & & & $14.73^{* *}$ & 2 & \\
\hline Noneloccasional & 2.07 & .61 & $11.44^{* *}$ & 1 & 7.93 \\
\hline Other/frequent & 1.45 & .71 & $4.12^{*}$ & 1 & 4.26 \\
\hline
\end{tabular}

Interaction between contact with the aggressor and thought about going

$6.40^{*} \quad 2$

back with him

\begin{tabular}{cccccc} 
None-occasional / going back & -1.02 & 1.72 & .35 & 1 & .362 \\
Other-frequent / going back & -4.76 & 2.31 & $4.24^{*}$ & 1 & .009 \\
\hline Model & & $X^{2}$ & $d f$ & \\
\hline Likelihood ratio & & $100.90^{*}$ & 2 & \\
Hosmer \& Lemeshow & .114 & 2 & \\
\hline
\end{tabular}

$R^{2}$ Cox \& Snell $=.128 ; R^{2}$ Nagelkerke $=.231$

${ }^{*} p<.05,{ }^{* *} p<.01$ 


\section{$\underline{\text { Short biographical note for authors }}$}

María García-Jiménez is a Ph.D. student at the Department of Experimental Psychology of the University of Seville. Her research focuses on the women's process of liberation from intimate partner violence and their experiences in the legal system. She holds a grant to develop her research funded by the Ministry of Education, Culture, and Sports of the government of Spain (FPU15/00373).

M. Jesús Cala-Carrillo, Ph.D., is Associate Professor at the Department of Experimental Psychology of the University of Seville. Since April 2012 to February 2016 she was Delegate of the Chancellor for Equality at the same University. In 2015 she was given the Meridiana Award by the Andalusian Regional Government for her initiatives against gender-based violence. She is a member of the Interdisciplinary Seminar on Women Studies of the University of Seville (SIEMUS) and her research lines are focused on intimate partner violence victims' recovery process and reasons why women disengage from legal proceedings, having led some projects on these topics.

M. Eva Trigo, Ph.D., is Associate Professor at the Department of Experimental Psychology of the University of Seville since 2002. Her research interests include methodological issues, innovations in teaching, and gender studies. She is a member of the Spanish Association of Methodology of the Behavioral Sciences (AEMCCO) and the Interdisciplinary Seminar on Women Studies of the University of Seville (SIEMUS). Since 2016 she serves as Director of the Office for Development Cooperation of the University of Seville. 